\title{
A DSP ACCELERATION FRAMEWORK FOR SOFTWARE-DEFINED RADIOS ON X86_64
}

\author{
Georgios Georgis, Alexios Thanos, Marcin Filo, Konstantinos Nikitopoulos \\ 5G Innovation Centre, Institute for Communication Systems, University of Surrey, Guildford, UK
}

\begin{abstract}
This paper presents a DSP acceleration and assessment framework targeting SDR platforms on x86_64 architectures. Driven by the potential of rapid prototyping and evaluation of breakthrough concepts that these platforms provide, our work builds upon the wellknown OpenAirInterface codebase, extending it for advanced, previously unsupported modes towards large and massive MIMO such as non-codebook-based multi-user transmissions. We then develop an acceleration/profiling framework, through which we present finegrained execution results for DSP operations. Incorporating the latest SIMD instructions, our acceleration framework achieves a unitary speedup of up to $10 \times$. Integrated into OpenAirInterface, it accelerates computationally expensive MIMO operations by up to $88 \%$ across tested modes. Besides resulting in a useful tool for the community, this work provides insight on runtime DSP complexity and the potential of modern x86_64 systems.
\end{abstract}

Index Terms - SDRs, parallel processing, SIMD, MIMO

\section{INTRODUCTION}

Software-Defined Radios (SDRs) have been nothing short of a breakthrough in making wireless systems research accessible. Having programmable platforms in place of an inflexible microchip, brought versatility and modularity via commercial off-the-shelf (COTS) devices in the form of $2 \times 2$ transceiver slices [1, 2, 3]. Their modular design allows platform components to be added transparently and with reduced effort. Combined with in-house software/firmware development, one can then conduct otherwise prohibitively expensive research into wideband and diverse transmissions, multiple-input multiple output (MIMO) configurations, new antenna designs and DSP optimization.

Even though baseband complexity based on SDRs has been studied in the past, up to now this has mostly been addressed on a theoretical basis, considering application-specific processors and architectures that can be considered slightly outdated for future wireless standards $[4,5]$. Hybrid programmable architectures are gaining ground due to their reconfigurability and shorter development cycle [6]. Especially with the arrival of the AVX512 instruction set, enabling 512-bit Single Instruction Multiple Data (SIMD) support in General-Purpose Processors (GPPs), the latter have become an attractive and powerful option for rapid prototyping [7]. In this direction, a number of GPP-based LTE stack solutions have surfaced, each one with a different roadmap and business model $[8,9,10,11]$. Among those, OpenAirInterface (OAI) is the most feature-rich (i.e., open-source, functional split support, non-realtime emulation, UE implementation, core network). OAI can be used to

This work was supported through UK's DCMS Testbeds and Trials projects "AutoAir" and "AutoAir Phase 2". The Authors would also like to thank the members of the University of Surrey's 5G Innovation Centre (http://www.surrey.ac.uk/5GIC) for their support. deploy a low-cost 3GPP cellular network using COTS SDRs and standard linux-based PCs. OAI's large developer community and the effort of multiple partners renders this the most popular and advanced publicly-available SDR platform. Despite OpenAirInterface leading the open-source competition, important functionalities are still incomplete or missing; e.g., multilayer and non-codebookbased transmission schemes in the case of $4 \mathrm{G}$, with $5 \mathrm{G}$ lacking even a bidirectional link. Of similar importance for a GPP-based Radio Access Network (RAN) is supporting advanced SIMD instructions. OAI takes limited advantage of newer instruction sets, as these were originally unsupported by the hardware upon its release and has only recently begun considering such features to improve performance [12]. Assessment of these aspects in GPP SDRs has been up to now very limited and restricted to the inherently supported modes $[13,14,15]$. This has left "uncharted territory" between plain software execution and hardware-assisted offloading, i.e., a feature that up to now has been considered inevitable for all computationally expensive operations. Evaluating GPP performance in the context of SDR acceleration is thus crucial, as a means to explore this territory.

Aiming to address these shortcomings, in this work we present: a) an SIMD acceleration toolbox for DSP operations, supporting AVX2 and AVX512 instructions, b) a software-accelerated linear precoder/detector supporting large MIMO transmissions, c) an acceleration / assessment framework for Orthogonal Frequency Division Multiplexing (OFDM) operations and d) a fine-grained profiling framework. To the best of our knowledge, it is the first time in the open literature that an SDR acceleration framework has been presented and profiled to such an extent. We also note our contributions enabling advanced, previously unsupported features within the stack of OpenAirInterface; dynamic beamforming and multi-user MIMO (MU-MIMO) for time-division duplexing (TDD). Section 2 describes our acceleration/profiling framework, Section 3 provides experimental results and Section 4 concludes.

\section{DSP ACCELERATION FRAMEWORK}

Our acceleration and profiling framework is transparent to the underlying 3GPP release, the transmission mode and the system's dimensionality. To highlight the impact of our contributions, we target MIMO operations, extending OAI with features previously unsupported. We added all necessary signaling for multi-layer non-codebook based precoding (changes spanning up to the Radio Resource Control (RRC) protocol). We introduce a new scheduler supporting up to four users over the same resources in both uplink (UL) and downlink (DL). Finally, we introduce support for the necessary baseband operations: a) channel estimation based on demodulation (UE-specific) reference signals (DMRS), b) modulation/demodulation, c) rate (de)matching and d) precoding/detection. Figure 1 illustrates the physical layer (L1) flow, highlighting optimized/extended features. Sub-blocks indicate the granularity of our profiling framework and grey blocks depict modules benefiting 


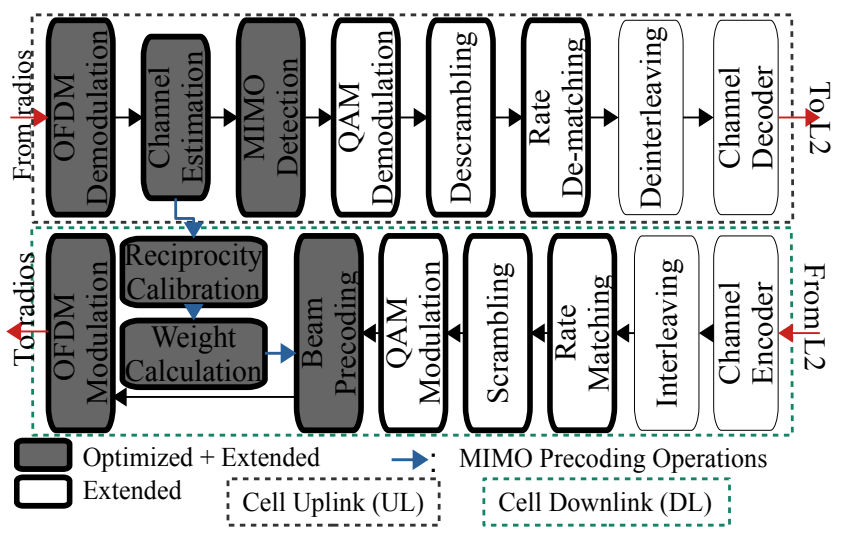

Fig. 1: Extended and optimized procedures in OAI's L1 flow.

by our acceleration framework. Targeting reusability, we currently avoid focus on standard-specific error correction.

\subsection{SIMD Acceleration}

OAI relies on fixed-point (i.e., integer) arithmetic throughout its L1 procedures [16], coded via low-level intrinsics for 128-bit SIMD (SSE) instructions (apart from Fourier transforms which use AVX2). Complemented by 16 bits per fixed-point I/Q sample, this allows processing up to 4 complex values within one 128-bit register. We introduce an SIMD acceleration framework which: a) supports SSE, AVX2 and AVX512 datapaths, b) focuses on MIMO operations, c) targets reusable DSP functions and d) supports series lengths $N$ not evenly divisible by the SIMD vector size $v s$ (i.e., $N \nmid v s$ ).

OpenAirInterface's physical layer DSP functions operate on complex time series between vectors and scalars. Our framework maintains the use of intrinsics for finer control over automatic compiler vectorization [17]. Regarding scalar/vector-vector addition and scalar-vector multiplication, we extend the vector width and the loop stride to enable the wider register instruction sets. In these cases, most of the SSE intrinsics have an AVX2/512 counterpart with a similar set of micro instructions.

OAI's complex multiplication and rotation though revolve around the _mm_madd_epil6 intrinsic (i.e., pmaddwd instruction) which horizontally multiplies and adds two 128-bit vectors across 16-bit boundaries. Just to use this instruction, OAI's complex rotation performs non-contiguous input memory accesses, creating a significant penalty which is aggravated in wider SIMD instructions. Since OAI's version creates 32-bit intermediate results, two 128-bit vectors are required to hold 4 complex numbers. We target elimination of horizontal operations, taking into account OAI's 16-bit representation and shifts. By initially replicating real and complex elements, we merge the pmaddwd, psrad, punpckldw and packssdw operations into the pshufb, pmulhrsw and paddsw instructions which maintain a 16-bit output. This generates fewer and lower latency instructions [18], having significant impact for a loop that is executed $\frac{N_{o f d m}}{v s} \cdot\left(N_{a n t}^{c e l l}\right)^{2} \cdot N_{\text {syms }}^{s f}$ times per subframe (e.g., the multadd_cpx_vector and apply/remove_7_5_kHz functions written similarly [19]). $N_{\text {ofdm }}$ denotes the number of samples in an OFDM symbol, $v s$ the SIMD vector size in samples, $N_{a n t}^{\text {cell }}$ the number of cell antennas and $N_{s y m s}^{s f}$ the subframe's length in OFDM symbols.

Due to a non-fixed arithmetic shift and the requirement for 32-bit accumulation, the above optimizations are not applicable to OAI's complex dot product. In that case and for the final AVX2 summation we employ the permute $2 \times 128$ si256 intrinsic, extracting the output via extract_epi32. In the case of AVX512, we employ reduce_add_epi32 and packs_epi32, manually composing the 32-bit output. We also eliminate any leftover MMX 64-bit instructions which incur an additional penalty requiring to empty the MMX state register at the end.

\subsubsection{Accelerated Zero Forcing precoding/detection}

A well-known linear method in large and massive MIMO research, [20, 21], Zero Forcing (ZF) can achieve high spectral efficiency gains when $N_{\text {ant }}^{B S} \gg N_{\text {layers }}$ (i.e., the number of layers). While considered to be simple-at least algorithmic-wise, due to the complex matrix multiplications and inversions involved, it has non-trivial computational and storage complexity, polynomially increasing with $N_{\text {ant }}^{\text {cell }}$ and $N_{\text {layers }}$. Our software ZF precoding/detection module supports AVX2/512 intrinsics, operating in parallel on a two-dimensional grid of antennas and resource elements. Our module is highly-configurable in terms of $N_{R E}$ (i.e., active resource elements), supporting up $N_{\text {ant }}^{\text {cell }}=N_{\text {layers }}=16$ in this instance. Both detection/precoding assume distinct channel matrices per subcarrier. Although $N_{s y m s}^{s f}$ is configurable, channel inversions are assumed to be performed once per subcarrier and group of OFDM symbols within a scheduling unit (e.g., $14 / 28$ symbols for $15 / 30 \mathrm{kHz}$ spacing [22]). Our precoder/detector operates on 16-bit fixed-point input/output performing single-precision calculations internally.

\subsubsection{OFDM subframe-based processing}

OFDM operations consist of discrete fourier transforms ( $d f t$ ), an optional shift of the zero-frequency component to the center of the spectrum (4G uplink), magnitude normalization and cyclic prefix removal/addition [22]. Particularly in large MIMO systems, these operations have a fixed yet significant complexity [23]. We developed a flexible subframe processing framework with modular $d f t$ kernels chosen at compile time. While $d f t$ acceleration libraries do exist: a) FFTW [24], Intel's b) MKL [25] and c) IPP [26], there has-to the best of our knowledge-been no aggregate evaluation of them in a 3 GPP context for SDRs [12]. Our OFDM framework allows the runtime definition of physical resource blocks $N_{R B}$, slots to schedule, $N_{a n t}^{\text {cell }}, \mu$ for subcarrier spacing [22] and repetitions for averaging.

\subsubsection{OAI integration}

All enhancements were added via preprocessor definitions allowing compile-time SIMD choice per function on all x86_64 platforms. We rewrote OAI's allocation functions to employ posix_memalign instead of the deprecated memalign, to align the allocated buffers according to vector size boundary requirements [17]. Up to now, OAI only considered data series lengths $N$ to be evenly divisible by the SIMD vector size ( $v s \mid N)$. While this works in the vast majority of the SSE cases, it will lead to segmentation faults when $v s \nmid N$ (e.g., for wider $v s$ ). To make our framework more robust, we include per function and per $v s$ length checking. A main loop processes data up to the point where its length is evenly divisible by vs. Remaining samples are then copied into an aligned static array whose length equals to $v s$. The vector pointers are then reinitialized to point into the array. This guarantees execution of the same intrinsics as those in the main function loop on an input with aligned boundaries. Our precoding/detection is integrated via a custom header/wrapper and a statically linked library. Finally, we note that since all accelerated 


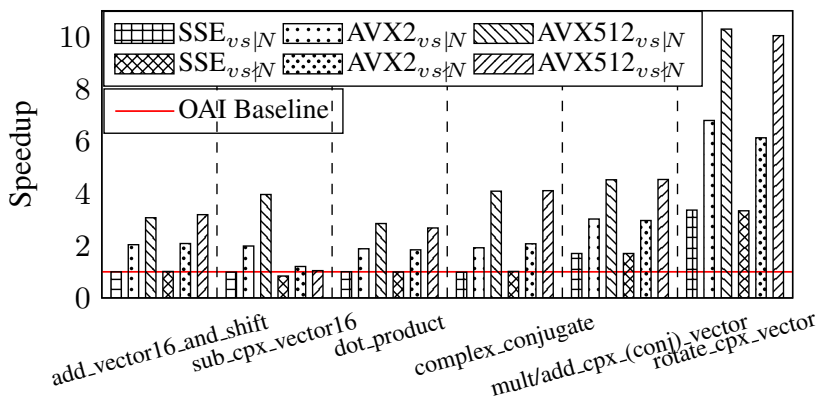

Fig. 2: Proposed DSP toolset speedup v. OAI's SSE baseline (red line, Intel Core i9-7980xe, $b \mid a$ denotes $a$ divisible by $b$ ).

$d f t$ libraries perform floating-point arithmetic, we developed SIMD conversion functions (including length checking). We keep a similar function interface to that of OAI's $d f t$ s and employ preprocessor definitions whithin the full L1 stack for compile-time $d f t$ library choice. The latter is also statically linked to the OpenAirInterface binaries.

\subsection{Profiling Framework}

Based on OAI's time-stamping mechanism (Time Stamp Counter, TSC) for Intel GPPs, we introduce a dedicated performance counter per L1 DSP function. Since most of these functions are executed several times within a radio frame, our performance counter also keeps track of the total number of function calls and subsequently calculates mean and minimum/maximum execution times. Our modifications allow multiple levels of granularity, from a single physical layer operation to aggregate, per subframe assessment. Hence, we can -in a streamlined/automated manner- get an accurate assessment of L1 procedures and explore the effect of different transmission modes and MIMO dimensions on execution time.

\section{ASSESSMENT METHODOLOGY AND RESULTS}

We note that generalizing functionality (e.g., bandwidths, 3GPP releases) within a full stack requires significant development effort and as such, for proof of concept we present full stack (i.e., within the context of OAI) measurements for a bandwidth of $5 \mathrm{MHz}$ (i.e., $N_{R E}=300$ ). Nevertheless, tests and results presented outside OAI's context are not bandwidth-limited. We profile execution on an Intel Core i9-7980XE, i.e., a representative of modern GPPs supporting AVX512 instructions. The processor operates at $2.6 \mathrm{GHz}$ running CentOS 7.6 with linux kernel 5.3 and 64GB of DDR4 RAM running at $2666 \mathrm{MHz}$. Intel's hyperthreading, turbo and low-power states were all switched off. We employ versions 3.3.3 for FFTW, 19 update 4 for Intel's libraries and 8.3.1 for the gcc/g++ compilers ${ }^{1}$.

\subsection{Tests outside OAI's context}

We created unitary $\mathrm{C}$ testbenches with runtime-configurable fixedpoint input range, data size and iterations. All functions were executed for $N=4096$ randomized complex samples (i.e., $a+j b$ ) with $a, b$ uniformly distributed in $[-1,1)$ and execution time averaged over $10^{6}$ iterations. For our precoder/detector we compare AVX2/512-optimized execution with that of a plain C model. Our

\footnotetext{
${ }^{1}$ Compilation flags used: $-\mathrm{O} 3$ - march=skylake-avx512 - mtune=skylakeavx512 -mprefer-vector-width=512 -fvect-cost-model=unlimited.
}

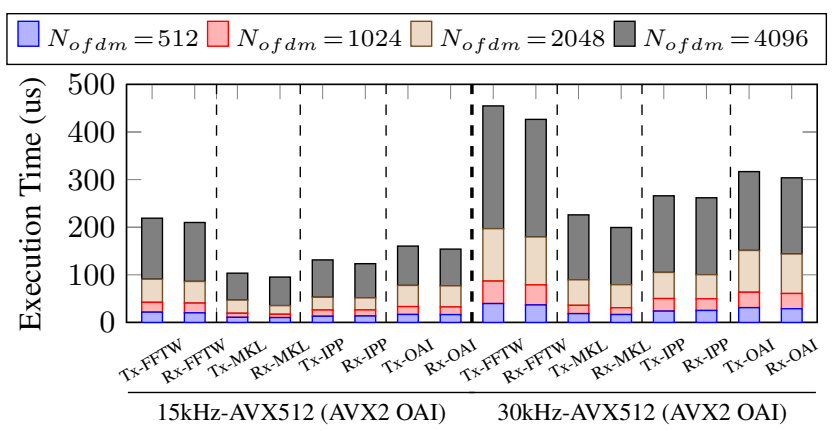

Fig. 3: OFDM Rx/Tx subframe execution $\left(N_{a n t}^{B S}=1\right)$.

testing scenarios assume $N_{\text {ant }}^{\text {cell }} \in\{2,4,8,16\}$ and $N_{\text {layers }} \in$ $\{2,4\}$. We measure subframe-based (i.e., $N_{\text {syms }}^{s f}=14$ ), singlethreaded execution times, corresponding to $N_{R E}=1272$ (i.e., $20 \mathrm{MHz}$ bandwidth [27]). Regarding OFDM assessment, initialization is performed once at the start of each execution. The term Tx/Rx subframe respectively corresponds to downlink/uplink only subframes. We showcase averaged results for $N_{\text {ofdm }} \in\{512$, 1024, 2048, 4096\}, encompassing $N_{R E} \in\{300,624,1272$, $3240\}$ and $N_{R E} \in\{288,612,1272,3276\}$ at $\{5,10,20,50\}$ and $\{10,20,40,100\} \mathrm{MHz}$ bandwidth for 15 and $30 \mathrm{kHz}$ subcarrier spacing respectively [27]. We note that all enhancements maintain or surpass the original quantization performance.

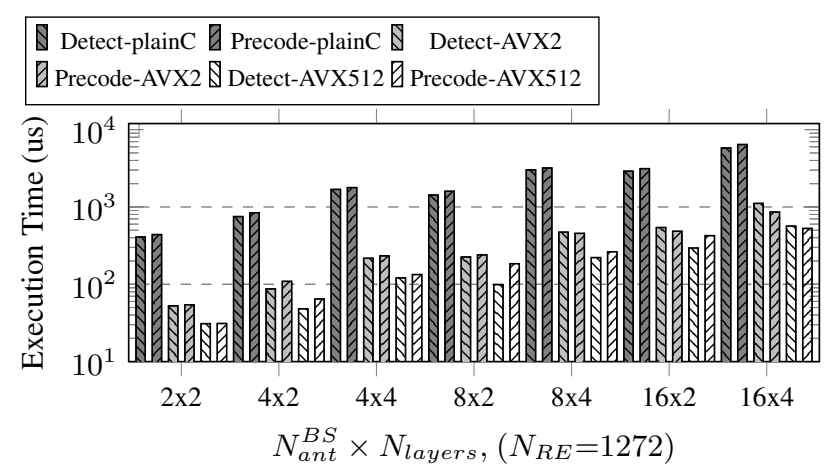

Fig. 4: Proposed Detector/Precoder: Plain C v. AVX2/512.

Figure 2 shows unitary speedup (and execution penalty in the cases where $v s \nmid N=4095$ ) for the most characteristic functions/ function groups. Achieved gains are within the sublinear region as expected. In particular, we observe average AVX2 speedups of $1.9 \times$ and average AVX512 speedups of $2.9-3.9 \times$ for functions which haven't been significantly redesigned (e.g., add_vector16_and_shift, sub_cpx_vector16). In the case of scalar/vector-vector multiplication, our optimisations accelerate OAI's out-of-the-box execution by $1.7-3.4 \times$, rising up to $4.5-10.3 \times$ (using SSE and AVX512 respectively). Penalty for $v s \nmid N$ v. $v s \mid N$ is generally negligible apart from complex rotation with AVX2 instructions $(6.2 \times$ v. $6.8 \times)$ and vector subtraction with AVX512 instructions (1.0× v. $3.9 \times)$ - the latter potentially having a small impact only on user-equipment-side.

Single-antenna results in Fig. 3 display that as expected, OFDM subframe execution time nearly doubles with subcarrier spacing. Intel's MKL surpasses the performance of all other dft libraries, the gain being more pronounced in higher bandwidths (e.g., 15\% faster v. IPP, $28 \%$ faster v. OpenAirInterface and $50 \%$ faster v. FFTW 

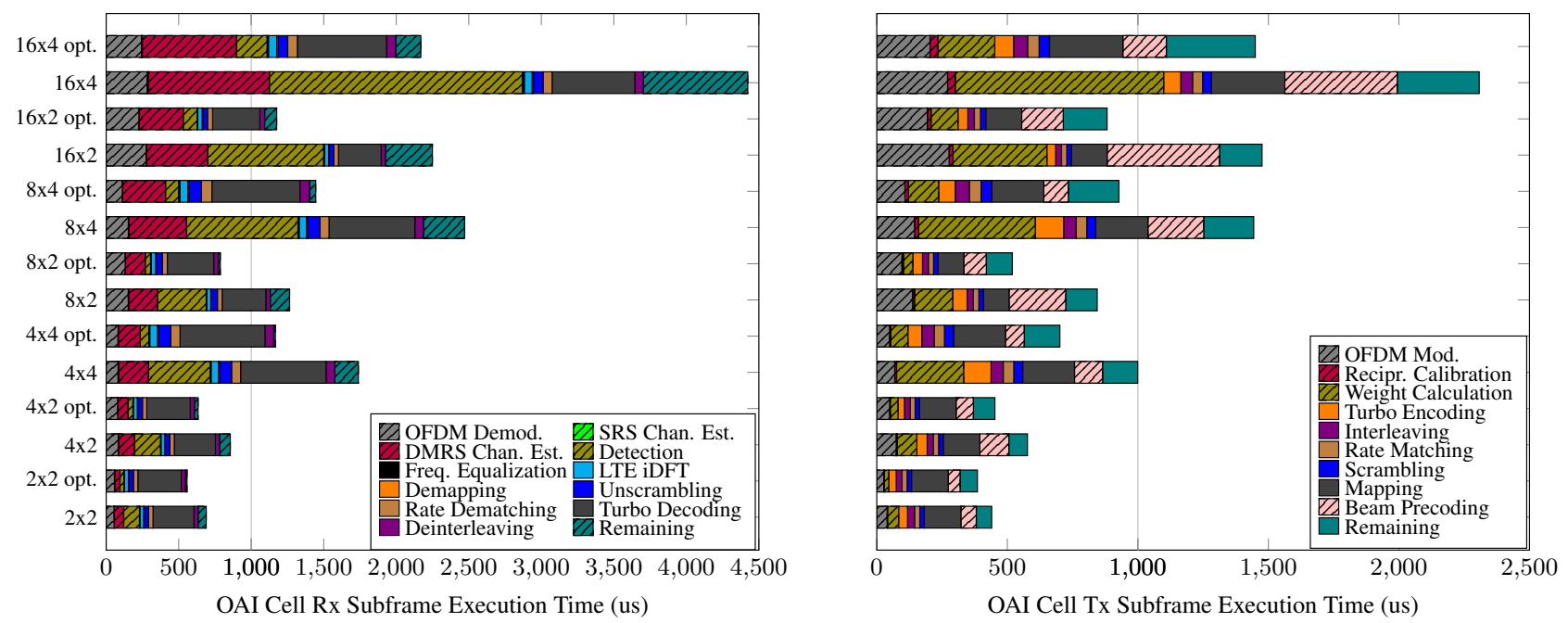

Fig. 5: Integrated L1 profiling: multi-user MIMO Rx/Tx subframe procedures pre/post- proposed optimizations $\left(N_{R E}=300\right)$.

for $N_{o f d m}=4096$ and Tx subframes at $30 \mathrm{kHz}$ spacing). Its performance is followed by the AVX512-enhanced IPP and OAI's libraries, the latter only supporting AVX2 (though still compiled and tested with the flags listed in this section). Regarding Zero Forcing MIMO detection/precoding, AVX512 surpasses AVX2 by $1.89 \times / 1.59 \times$ respectively, attaining an order of magnitude benefit when compared to non-vectorized $\mathrm{C}$ code. (Fig. 4). Execution time stays below $600 \mu \mathrm{s}$ even for the challenging $16 \times 4$ MU-MIMO case.

\subsection{Integrated OAI tests}

We measure cell execution (i.e., the most computationally demanding) via OpenAirInterface's emulation mode. The latter simulates only the radio equipment and the channel (handled by separate processes), the remaining protocol stack running as it would on a realtime system. We present single-threaded physical layer results with any extra workers disabled in the configuration files. We note that our tests on OpenAirInterface's current threading architecture showed marginal gains on Frequency Division Duplexing (FDD) schemes only. The cell was set to operate at $3.5 \mathrm{GHz}$ using Time Division Duplexing (with $50 \%$ uplink/downlink slot allocation) in monolithic mode (i.e., frontend/baseband processing taking place on the cell). For single host execution, we configure the cell and userequipments' packet-based interfaces in internal loopback. Following the initialization of all processes within the stack and the establishment of cell to user equipment connection (via the Random Access Channel-RACH procedure), we generate bidirectional (i.e., uplink and downlink) data traffic via iPerf [28]. We gather results over the course of 60 minutes for the aforementioned MIMO configurations.

Figure 5 presents results integrating our profiling contributions and our AVX512-accelerated framework (denoted with "opt", bars shaded with dashed lines depicting affected operations). We compare against OAI's default SIMD datapath, except for Zero Forcing precoding/detection which employs non-vectorized code as a baseline (i.e., there was no pre-existing code for this operation). Listed procedures involve uplink (i.e., Rx, Fig. 5-left) and downlink shared channels (i.e., Tx, Fig. 5-right), for physical layer operations scheduled on a subframe basis (i.e., $N_{s y m s}^{s f}=14$ [22]). “(De)mapping” includes layer (de)mapping and QAM (de)modulation, while "Remaining" refers to outstanding physi- cal channels and control/software overheads.

Regarding runtime execution of Rx subframes, MIMO detection benefits by more than $75 \%$ across all tested cases. SRS/DMRS channel estimation respectively benefit by $47 / 30 \%$, the latter affecting total subframe execution significantly more than the former (up to $\frac{1}{5}$ of the total Rx execution time in $16 \times 4$ non-optimized MUMIMO - Fig. 5-left). Remaining operations also benefit by $80 \%$ on average. Total $\mathrm{Rx}$ subframe execution speedup ranges from $1.17 \times$ $(2 \times 2 \mathrm{MU}-\mathrm{MIMO})$ to $1.95 \times$ for $16 \times 4 \mathrm{MU}-\mathrm{MIMO}$, effectively reducing execution time from 4.4 to approximately $2.2 \mathrm{~ms}$ (Fig. 5-left). Tx precoding can take up to half of the total execution time in high order MU-MIMO (Fig. 5-right). On average, our optimisations accelerate Weight Calculation by $69 \%$ and Beam Precoding (i.e., weight application) by $48 \%$. Integrating MKL's $d f t$ libraries and our SIMD conversion functions incurs a $29 \%$ average OFDM modulation speedup. Average Tx subframe acceleration is up to $1.6 \times\left(N_{a n t}^{B S}=16\right)$.

While exceeding the $1 \mathrm{~ms}$ barrier breaks realtime execution, the opposite is not always true. Generalizing and quantifying all dependencies (front-end, radio and over-the-air latency, threading architecture), is well-beyond the scope of this work. Overall, we observe channel decoding to be the most expensive Rx operation, $9 \times$ more demanding compared to LTE's Turbo encoder (executed on an SSE datapath for OAI). DMRS channel estimation can be as demanding in $16 \times 4 \mathrm{MIMO}$, followed by OFDM procedures and detection/precoding. Results indicate that more pronounced gains should be expected in higher bandwidths.

\section{CONCLUSIONS}

This work presented an SIMD acceleration framework for SDRs. Harnessing AVX512, our contributions speed up unitary OAI SSE operations by up to $10 \times$ and more involved procedures by up to an order of magnitude compared to non-vectorized code. Following significant functional extensions to support advanced transmission schemes, our integrated contributions enhance OAI subframe execution by up to $1.95 \times$. Our regression testing/profiling framework facilitates assessment and highlights the potential/limits of modern x86_64 systems. Future work will focus on exploiting hardwareassisted offloading and multicore execution on top of our SIMD framework, as well as on channel coding. 


\section{REFERENCES}

[1] Ettus Research, "Ettus Research, a National Instruments Company," https://www.ettus.com/site/about, 2019.

[2] Fairwaves, "XTRX, The first truly embedded SDR," https:// www.crowdsupply.com/fairwaves/xtrx, 2019.

[3] Skylark Wireless, "Skylark Wireless, Broadband for the next billion," http://www.skylarkwireless.com/, 2019.

[4] Woh M., Lin Y., Seo S., Mudge T., and Mahlke S., "Analyzing the scalability of simd for the next generation software defined radio," in 2008 IEEE Int. Conf. on Acoust., Speech and Signal Process., March 2008, pp. 5388-5391.

[5] P. Westermann and H. Schröder, "On the scalability of simd processing for software defined radio algorithms," in 2010 Int. Conf. on Embedded Comput. Syst.: Architectures, Model. and Simul., July 2010, pp. 309-317.

[6] G. Sklivanitis, A. Gannon, S. N. Batalama, and D. A. Pados, "Addressing next-generation wireless challenges with commercial software-defined radio platforms," IEEE Commun. Mag., vol. 54, no. 1, pp. 59-67, January 2016.

[7] C. S. Anderson, J. Zhang, and M. Cornea, "Enhanced vector math support on the Intel AVX-512 architecture," in IEEE 25th Symp. on Comput. Arithmetic (ARITH), Jun. 2018, pp. 120124.

[8] Amarisoft, "LTE 100, a full software LTE solution," https: //www.amarisoft.com/, 2019.

[9] I. Gomez-Miguelez, A. Garcia-Saavedra, P. D. Sutton, P. Serrano, C. Cano, and D. J. Leith, "srsLTE: An open-source platform for LTE evolution and experimentation," in Proc. 10th ACM Int. Workshop Wireless Netw. Testbeds, Exp. Eval. Characterization. 2016, WiNTECH '16, pp. 25-32, ACM.

[10] N. Nikaein, R. Knopp, F. Kaltenberger, L. Gauthier, C. Bonnet, D. Nussbaum, and R. Ghaddab, "Demo: OpenAirInterface: An open LTE network in a PC,' in Proc. 20th Annu. Int. Conf. Mobile Comput. and Netw. 2014, MobiCom '14, pp. 305-308, ACM.

[11] openLTE, "OpenLTE; an open source implementation of the 3GPP LTE specifications," https://sourceforge.net/projects/ openlte/, 2019.

[12] Shah S., "Proposed features for OpenAirInterface," https:// trello.com/c/cbhbyc46/94-performance-improvements, 2019.

[13] C. Y. Yeoh, M. H. Mokhtar, A. A. A. Rahman, and A. K. Samingan, "Performance study of lte experimental testbed using openairinterface," in 2016 18th Int. Conf. on Adv. Commun. Technol. (ICACT), Jan 2016, pp. 617-622.

[14] Z. Geng, X. Wei, H. Liu, R. Xu, and K. Zheng, "Performance analysis and comparison of gpp-based sdr systems," in 2017 7th IEEE Int. Symp. on Microw., Antenna, Propag., and EMC Technol. (MAPE), Oct 2017, pp. 124-129.

[15] Gringoli F., Patras P., Donato C., Serrano P., and Grunenberger Y., "Performance assessment of open software platforms for 5g prototyping," IEEE Wireless Commun., vol. 25, no. 5, pp. 10-15, October 2018.

[16] Labrosse J. J., Embedded Systems Building Blocks, Complete and Ready-to-Use Modules in C, R\&D Technical Books, 1995.

[17] Voss M., "Topics in loop vectorization,” https://www.cs.utexas. edu/ pingali/CS377P/2019sp/lectures/vectorization-voss.pdf, 2018.
[18] Intel, "Intel Intrinsics Guide," https://software.intel.com/sites/ landingpage/IntrinsicsGuide/, 2019.

[19] Eurecom, “openairinterface5G git repository," https://gitlab. eurecom.fr/oai/openairinterface 5g, 2019.

[20] C. Shepard, H. Yu, N. Anand, E. Li, T. Marzetta, R. Yang, and L. Zhong, "Argos: Practical many-antenna base stations," in Proc. 18th Annu. Int. Conf. Mobile Comput. and Netw. ACM, 2012, pp. 53-64.

[21] S. Malkowsky, J. Vieira, L. Liu, P. Harris, K. Nieman, N. Kundargi, I. C. Wong, F. Tufvesson, V. Öwall, and O. Edfors, "The world's first real-time testbed for massive MIMO: Design, implementation, and validation," IEEE Access, vol. 5, pp. 90739088, 2017.

[22] 3GPP, "Physical Channels and Modulation," Technical Report (TR) 36.211, 3rd Generation Partnership Project (3GPP), Dec. 2018, Version 15.4.0.

[23] H. S. Stone, "Parallel processing with the perfect shuffle," IEEE Trans. Comput., vol. C-20, no. 2, pp. 153-161, Feb. 1971.

[24] Frigo M. and Johnson S. G., "FFTW," http://www.fftw.org/, 2018.

[25] Intel, "Intel@ Math Kernel Library," https://software.intel. com/en-us/mkl, 2018.

[26] Intel, "Intel@ Integrated Performance Primitives," https:// software.intel.com/en-us/intel-ipp, 2018.

[27] 3GPP, "Base Station (BS) radio transmission and reception," Technical Report (TR) 36.104, 3rd Generation Partnership Project (3GPP), Dec. 2018, Version 15.4.0.

[28] "iPerf - The TCP, UDP and SCTP network bandwidth measurement tool," https://iperf.fr/, 2019. 